\title{
AVALIAÇÃO DA ELETROFLOTAÇÃO NO TRATAMENTO DE ÁGUA PRODUZIDA DE PETRÓLEO
}

\author{
G. S. CUNHA ${ }^{1}$ e A. J. B. DUTRA ${ }^{2}$ \\ Laboratório de Eletroquímica Aplicada - Universidade Federal do Rio de Janeiro \\ gustavoscunha@metalmat.ufrj.br ${ }^{1}$ - adutra@metalmat.ufrj.br ${ }^{2}$
}

Artigo submetido em novembro/2013 e aceito em fevereiro/2014

DOI: http://dx.doi.org/10.15628/holos.2014.1821

\section{RESUMO}

Os processos de extração, transporte e refino de petróleo geram águas residuárias com uma gama considerável de efluentes orgânicos e inorgânicos, tais como hidrocarbonetos, fenóis, metais pesados, amônia, entre outros. Por isso, faz-se necessário o tratamento destes efluentes antes de lançá-lo ao meio ambiente ou reutilizá-lo em alguma etapa de produção. Devido a elevada eficiência e compactabilidade, as tecnologias eletroquímicas se mostram promissoras no tratamento de efluentes, especialmente a eletroflotação. O objetivo deste trabalho é aplicar a eletroflotação como alternativa ao tratamento de amostras de água produzida de petróleo, visando principalmente a remoção de matéria orgânica. Foram comparados os valores de Demanda Química de Oxigênio (DQO) antes e depois do ensaio em coluna. Utilizou-se, como catodo, aço inox, e, como anodo, titânio revestido com óxido de rutênio. As tensões aplicadas variaram entre 2,5 e $5,5 \mathrm{v}$. Os tempos de flotação foram 10, 20, 30, 40 e $50 \mathrm{~min}$. Os resultados apontaram uma considerável queda da carga contaminante após 50 minutos de flotação, sob tensões de cerca de 5,5 v.

PALAVRAS-CHAVE: efluentes, eletroquímica, flotação

\section{EVALUATION OF THE ELECTROFLOTATION ON PRODUCED WATER TREATMENT}

\begin{abstract}
The processes of extraction, transportation and refining of oil generate wastewater with a considerable range of organic and inorganic waste, such as hydrocarbons, phenols, heavy metals, ammonia, among others. Therefore, it is necessary to treat such wastewaters before releasing it to the environment or reuse it in some stage of production. Due to the high efficiency and compactability, electrochemical technologies have shown to be an interesting alternative in the treatment of effluents, particularly the electroflotation. The objective of this work was to test electroflotation as an
\end{abstract}

alternative to the treatment of original samples of produced water from oil, aiming at the removal of organic matter. The Chemical Oxygen Demand (COD) values before and after the test column were reported. It was used as cathode, stainless steel, and as anode, titanium coated with ruthenium oxide. The applied voltages varied between 2.5 and $5.5 \mathrm{v}$. The flotation times were 10, 20, 30,40 and $50 \mathrm{~min}$. Results shown a considerable decrease in contaminant load after 50 minutes of flotation under voltages around 5,5 v.

KEYWORDS: wastewater, electrochemistry, flotation 


\section{INTRODUÇÃO}

A poluição das águas vem se agravando cada vez mais e tem despertado a atenção do mundo. Porém, apesar das iniciativas globais em se evitar desastres de contaminação, esses eventos continuam ocorrendo, pois a maior parte dos processos industriais são muito poluentes e apresentam baixa eficiência de conversão, gerando resíduos que muitas vezes não são tratados de forma adequada antes do desague no meio ambiente (QUINETTI, 2005).

Os processos de produção de petróleo - extração, transporte e refino - geram águas residuárias como efluente. A composição dessas águas pode variar muito, contendo alta concentração de óleo, elevada salinidade, sólidos suspensos, amônia, metais pesados, gases dissolvidos, microrganismos e outros compostos (BANDE et al., 2007).

No tratamento de efluentes gerados pela atividade petrolífera podem ser utilizados processos físicos, químicos, físico-químicos e biológicos, tais como o uso de separadores água-óleo, coagulação-floculação, lagoas de ativação, lodo ativado, filtros biológicos, processos oxidativos avançados e tecnologia eletroquímica (CERQUEIRA e MARQUES, 2011). De acordo com Ahmadum et al. (2009), os objetivos esperados no tratamento de água produzida são: Remover graxa e óleo disperso, remover compostos orgânicos solúveis, desinfecção, remover partículas suspensas e areia, remover sais dissolvidos, remover o excesso de dureza da água.

O tratamento eletroquímico é considerado um dos mais poderosos processos de oxidação utilizados no controle ambiental. Como vantangens, além da alta eficiência de remoção, este método pode ser usado em baixas temperaturas, seus equipamentos são simples e compactos, é de fácil manuseio e controle. O elétron é o principal agente oxidante evitando-se, assim, o uso de reagentes químicos que poderiam gerar sub-produtos tão ou mais poluentes quanto os de origem (KIM et al., 2002).

A eletroflotação é um processo que promove a adesão e flutuação de partículas até a superfície de um corpo d'água através da geração de microbolhas oriundas da decomposição eletrolítica da água (BANDE et al., 2007). As principais vantagens da eletroflotação em relação à flotação tradicional são a rápida taxa de remoção de partículas, o alcance simultâneo de flotação e coagulação, menor quantidade de lama formada e fácil instação e utilização (NAHUI et al., 2008). Na remoção de Demanda Química de Oxigênio (DQO), a eletroflotação atua na flotabilidade de gotículas de óleo presentes na água de processo. As reações de formação de bolhas de oxigênio e hidrogênio no anodo e no catodo, respectivamente, são mostradas a seguir:

$$
2 \mathrm{H}_{2} \mathrm{O} \rightarrow \mathrm{O}_{2}+4 \mathrm{H}^{+}+4 \mathrm{e}^{-}
$$

$$
4 \mathrm{H}_{2} \mathrm{O}+4 \mathrm{e}^{-} \rightarrow 2 \mathrm{H}_{2}+4 \mathrm{OH}^{-}
$$

Na eletroflotação, as bolhas de gás formadas durante a eletrólise são extremamente pequenas e uniformes, com diâmetros que podem chegar a $20 \mu \mathrm{m}$. Bolhas com diâmetros pequenos promovem aumento na eficiência de colisão entre bolhas e partículas e geram, assim, melhores resultados no processo. A variação da densidade de corrente como consequência da variação da tensão pode influenciar no tamanho de bolhas da eletroflotação. Quanto maior a 
densidade de corrente, menor será o tamanho de bolhas. Este fato está relacionado com a nucleação e crescimento de uma nova fase sobre o eletrodo (SANTOS e DUTRA, 2011).

Durante a eletroflotação, ocorre também oxidação da matéria orgânica. A oxidação eletroquímica pode ser dada de forma direta ou indireta. No processo direto, os poluentes são adsorvidos na superfície anódica e depois são destruídos por reações provocadas pela transferência de elétrons. No processo indireto, são gerados eletroquimicamente oxidantes fortes, como o hipoclorito e o cloreto, os quais destroem os contaminantes através de reações de oxidação. As reações que mostram a formação de espécies oxidantes em amostras de alta salinidade podem ser observadas abaixo (RAJKUMAR e PALANIVELU, 2004):

$2 \mathrm{Cl}^{-} \rightarrow \mathrm{Cl}_{2}+2 \mathrm{e}^{-}$

$\mathrm{Cl}_{2}+\mathrm{H}_{2} \mathrm{O} \rightarrow \mathrm{HOCl}+\mathrm{H}^{+}+\mathrm{Cl}^{-}$

$\mathrm{HOCl} \rightarrow \mathrm{H}^{+}+\mathrm{OCl}^{-}$

O objetivo desse trabalho foi avaliar a influência do tempo e da variação da tensão na remoção de DQO de efluentes da indústria petrolífera por meio de um processo de eletroflotação em coluna.

\section{MATERIAIS E MÉTODOS}

Inicialmente, foram quantificados alguns parâmetros do efluente, como: DQO, concentração de cloreto, $\mathrm{pH}$ e condutividade. Devido à alta salinidade do efluente, as medidas de DQO foram realizadas conforme o método proposto por FREIRE \& SANTANA JR. (1998). A concentração de cloreto foi determinada pelo método de Mohr e a condutividade e o pH foram quantificados pelo medidor de pH HANNA modelo HI255. Todos os experimentos foram feitos em duplicata.

\subsection{Quantificação de DQO}

\subsubsection{Solução digestora}

Após ter sido obtida a relação entre DQO e absorbância por calibração, uma solução digestora foi preparada através da dissolução de 5,1 g de dicromato de potássio, $70 \mathrm{~mL}$ de ácido sulfúrico e 41,65 g de sulfato de mercúrio em $250 \mathrm{~mL}$ de água destilada.

\subsubsection{Solução catalisadora}

Uma solução catalisadora foi preparada a partir da adição de 2,525 g de sulfato de prata em $250 \mathrm{~mL}$ de ácido sulfúrico. 


\subsubsection{Procedimento para medida}

Foram adicionados em tubos de ensaio, $2 \mathrm{~mL}$ de amostra do efluente previamente diluído, $1,2 \mathrm{~mL}$ de solução digestora e $2,8 \mathrm{~mL}$ de solução catalisadora. Para o branco, utilizou-se água destilada ao invés do efluente. Os tubos foram levados para digestão em um aquecedor HANNA $\mathrm{HI} 839800$ a $150^{\circ} \mathrm{C}$ por 2 horas. Após este tempo, foram resfriados em temperatura ambiente e armazenados em geladeira por 24 horas. Em seguida, foi utilizado um espectrofotômetro para a medida da absorbância em $600 \mathrm{~nm}$ de cada amostra em temperatura ambiente. Com a absorbância, pôde-se aplicar a curva de calibração e, então, encontrar os valores de DQO correspondentes.

\subsection{Medida da concentração de cloreto}

Dissolveu-se $8,5 \mathrm{~g}$ de nitrato de prata em $250 \mathrm{~mL}$ de água para o preparo de uma solução padrão de nitrato de prata $0,2 \mathrm{~mol} . \mathrm{L}^{-1}$. Misturou-se $15 \mathrm{~mL}$ de amostra do efluente com $25 \mathrm{~mL}$ de água e $1 \mathrm{~mL}$ de indicador (cromato de potássio) para ser titulada com a solução de nitrato de prata até mudança de cor de amarelo para marron avermelhado. $O$ volume gasto de titulante foi anotado para se calcular o teor de cloreto.

\subsection{Ensaios de eletroflotação}

Os ensaios de eletroflotação foram realizados em coluna de acrílico, como esquematizada na figura abaixo:

\begin{tabular}{|l|}
\hline Legenda \\
\hline A - Entrada do Efluente \\
\hline B - Saida para coleta de amostra \\
\hline C - Saida de Lodo \\
\hline D - Coluna de eletroflotação \\
\hline E - Reservatório de lodo \\
\hline
\end{tabular}

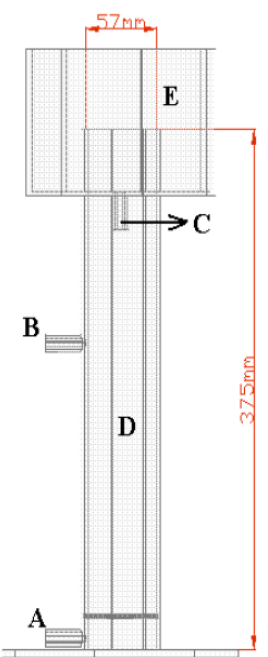

Figura 1 - Esquema da célula de eletroflotação

Utilizou-se aço inox como catodo e titânio revestido com óxido de rutênio como anodo. A escolha foi baseada em dados da literatura devido a grande capacidade de oxidação de matéria orgânica desse anodo em meio salino. Os eletrodos acoplados na parte inferior da coluna foram conectados a uma fonte de tensão e, realizaram-se experimentos em batelada variando a tensão em $2,5,3,5,4,5$ e 5,5 v. A cada 10 minutos foi coletada uma pequena quantidade de amostra do 
efluente para a análise da DQO. A duração total de cada ensaio de eletroflotação em uma tensão específica foi de 50 minutos.

\subsection{Cinética da reação}

Segundo Palanivelu e Rajkumar (2004), na oxidação indireta a taxa de remoção de DQO é proporcional à concentração do poluente e à concentração de cloro/hipoclorito. A equação abaixo mostra a cinética para a remoção de DQO:

$$
-\frac{\mathrm{d}}{\mathrm{dt}}[\mathrm{DQO}]=\mathrm{k}[\mathrm{DQO}]\left[\mathrm{Cl}_{2}\right]
$$

Durante a eletrólise, cloreto é convertido a cloro e/ou hipoclorito, dependendo do $\mathrm{pH}$, que após oxidar a matéria orgânica serão reduzidos a íon cloreto novamente. O processo, então, se repete rapidamente e, por isso, a concentração de cloro/hipoclorito é considerada constante e assume um modelo de pseudo primeira-ordem, conforme a equação abaixo:

$$
-\frac{d}{d t}[D Q O]=k \cdot[D Q O]
$$

Com a integração, tem-se:

$$
\ln \frac{[\mathrm{DQO}]_{\mathrm{t}}}{[\mathrm{DQO}]_{0}}=-\mathbf{k}^{\prime} \mathbf{t}
$$

Assim, pôde-se determinar os valores das constantes cinéticas a partir da inclinação da reta obtida nos gráficos de In [DQO $]_{t} /[D Q O]_{0}$ pelo tempo.

\subsection{Eficiência de Corrente e Consumo Energético}

Para o cálculo de eficiência de corrente (EC) e consumo energético (CE) foram utilizadas as seguintes equações, conforme Comninellis e Pulgarin (1991):

$$
\begin{aligned}
& \mathrm{EC}=\frac{\mathrm{FV}\left[\left(\mathrm{DQO}_{0}-\mathrm{DQO}_{\mathrm{t}}\right)\right]}{8 \mathrm{It}} \\
& \mathrm{CE}=\frac{\mathrm{UIt}}{1000 \mathrm{~V}}
\end{aligned}
$$

Onde $\mathrm{F}$ é a constante de Faraday, $\mathrm{V}$ é o volume da amostra ( $L$ para EC e $\mathrm{m}^{3}$ para CE), I é a corrente (A), t é o tempo (h), $\mathrm{U}$ é a tensão aplicada (v), $D Q O_{0}$ é a Demanda Química de Oxigênio no início do processo e $D Q O_{t}$ é a Demanda Química de Oxigênio após um tempo t de operação. 


\section{RESULTADOS E DISCUSSÃO}

As amostras utilizadas nessa pesquisa foram cedidas pelo terminal marítimo Almirante Barroso (Tebar/Transpetro), localizado em São Sebastião, São Paulo. O efluente é oriundo dos processos de armazenamento e transporte de petróleo e derivados e suas características iniciais são mostradas na tabela abaixo:

Tabela 1 - Características iniciais do efluente utilizado

\begin{tabular}{lc}
\hline DQO (mg. $\left.\mathrm{L}^{-1}\right)$ & 1178,7 \\
pH & 7,57 \\
Condutividade $\left(\mathrm{mS} . \mathrm{cm}^{-1}\right)$ & 60,33 \\
Concentração de cloreto $\left(\mathrm{mg} \cdot \mathrm{L}^{-1}\right)$ & 96486,7 \\
\hline
\end{tabular}

O elevado valor de DQO é justificado pela grande quantidade de contaminantes orgânicos. A alta condutividade tem relação com a concentração de cloreto, bastante significativa. Condutividades elevadas podem reduzir a tensão da célula devido à diminuição da resistência ôhmica do efluente. Além disso, em eletroflotação a salinidade promove diminuição no tamanho de bolhas dos gases formados, se comparado com processos com pouca ou nenhuma salinidade. A velocidade de ascensão das bolhas de diâmetros menores é baixa e, por isso, sobem lentamente para a superfície, tornando-se maior a possibilidade de colisão com gotículas de óleo, melhorando, desta forma, a remoção de carga orgânica.

A Figura 2 mostra a variação da DQO ao longo do tempo em diferentes tensões aplicadas.

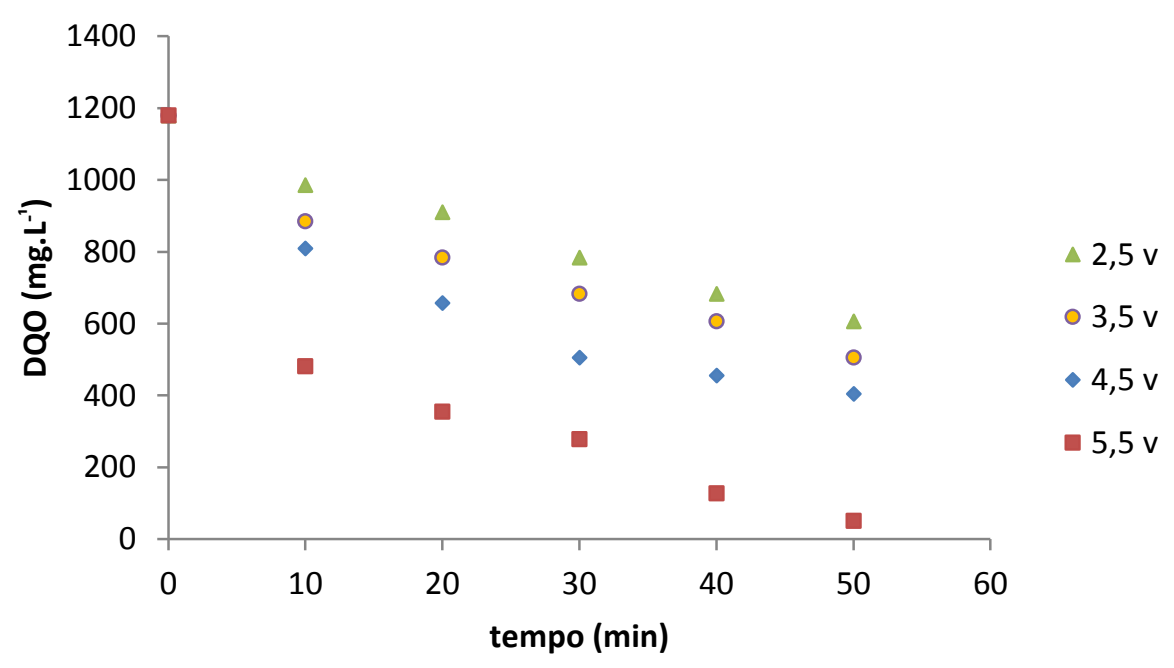

Figura 2 - Influência da tensão aplicada na concentração de DQO ao longo do tempo

Pode ser observado que quanto maior a tensão aplicada, maior será a remoção de matéria orgânica. A DQO inicial de $1179 \mathrm{mg} \cdot \mathrm{L}^{-1}$ foi reduzida para cerca de $602 \mathrm{mg} \cdot \mathrm{L}^{-1} \mathrm{em} \mathrm{2,5}$ ve para 50,5 mg. $\mathrm{L}^{-1} \mathrm{em} 5,5 \mathrm{v}$, após 50 minutos de eletroflotação, correspondendo a percentuais de remoção de 48,6 e $95,7 \%$ respectivamente. O aumento da tensão eleva a corrente, promove maiores taxas de oxidação eletroquímica, aumenta a taxa de geração de gases hidrogênio, oxigênio e cloro gerados nos eletrodos e diminui o tamanho de bolhas. Assim, há maior oxidação da matéria orgânica, pela maior quantidade de sub-produtos ativos formados, e mais gotículas de óleo são conduzidas à 
superfície pelas bolhas de gás. Assim como a tensão, o aumento do tempo influencia positivamente na diminuição do teor de contaminantes orgânicos. Remoções mais significativas são encontradas após 30 minutos de ensaio.

As constantes cinéticas foram obtidas por regressão linear, segundo o gráfico abaixo:

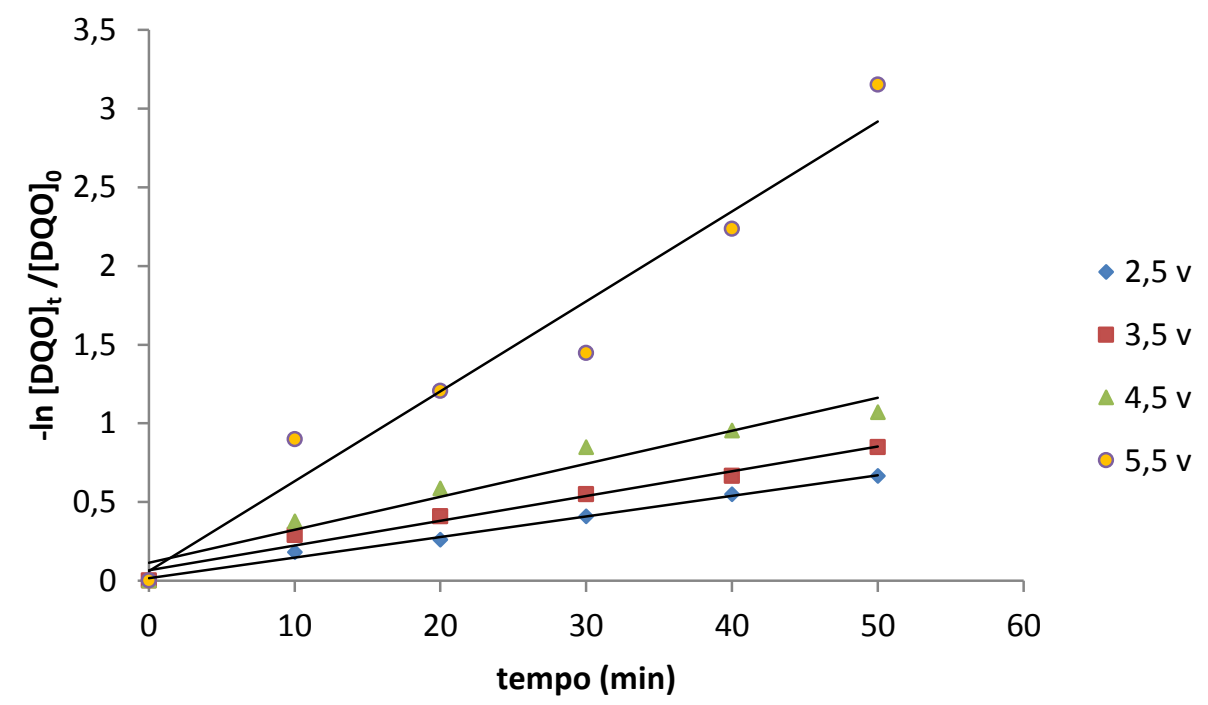

Figura 3 - Razão logarítimica das concentrações de DQO finais e iniciais em função do tempo

A Tabela 2 mostra a constante cinética, a eficiência de corrente e o consumo energético para cada tensão aplicada em 50 minutos de eletroflotação:

Tabela 2 - Influência da tensão aplicada na constante cinética, eficiência de corrente e consumo energético para remoção de DQO de efluentes de água de produção de petróleo.

\begin{tabular}{|c|c|c|c|c|c|}
\hline \multirow{2}{*}{$\begin{array}{c}\text { Tensão } \\
\text { aplicada } \\
\text { (v) }\end{array}$} & \multirow{2}{*}{$\begin{array}{l}\text { Constante } \\
\text { Cinética } \\
\mathbf{k}^{\prime}\left(\min ^{-1}\right)\end{array}$} & \multirow{2}{*}{$\begin{array}{c}\text { Coeficiente } \\
\text { de correlação }\end{array}$} & \multicolumn{2}{|c|}{$\begin{array}{c}\text { Eficiência de Corrente } \\
(\%)\end{array}$} & \multirow{2}{*}{$\begin{array}{l}\text { Consumo } \\
\text { Energético } \\
\left(\mathrm{kwh} / \mathrm{m}^{3}\right)\end{array}$} \\
\hline & & & $20 \mathrm{~min}$ & $50 \mathrm{~min}$ & \\
\hline 2,5 & $1,3.10^{-2}$ & 0,99 & 98,8 & 83,9 & 0,35 \\
\hline 3,5 & $1,6.10^{-2}$ & 0,98 & 28,1 & 19,1 & 2,58 \\
\hline 4,5 & $2,1.10^{-2}$ & 0,97 & 10,0 & 5,9 & 12,26 \\
\hline 5,5 & $5,7.10^{-2}$ & 0,97 & 9,4 & 5,1 & 25,27 \\
\hline
\end{tabular}

Em 2,5 v, a eficiência de corrente é elevada. À medida que aumenta-se a tensão, a eficiência de corrente alcança valores cada vez mais baixos. A eficiência de corrente também tende a diminuir ao longo do tempo. Já o consumo energético aumenta com o aumento da tensão. Dessa forma, é possível se obter valores ótimos de consumo energético, manipulando-se a tensão.

\section{CONCLUSÃO}

Através deste trabalho pode-se concluir que o aumento do tempo e da tensão influencia positivamente o processo de remoção de matéria orgânica. 95,7 \% de DQO foi removida aplicando- 
se 5,5 v em 50 minutos de operação. Processos em que se utilizam maiores tensões apresentam maior consumo de energia, porém, neste estudo, o custo energético foi relativamente baixo, o que poderia viabiliazar a técnica como alternativa no controle da poluição de águas contaminadas da indústria petrolífera.

\section{REFERÊNCIAS}

1. AHMADUN, F.R., PENDASHTEH, A., ABDULLAH, L.C., BIAK, D.R.A., MADAENI, S.S., ABIDIN, Z.Z. Review of technologies for oil and gas produced water treatment. Journal of Hazardous Materials, v. 170, issue 2-3, p. 530-551. 2009.

2. BANDE, R.M., PRASAD, B.B., MISHRA, I.M., WASEWAR, K.L. Oil field effluent water treatment for safe disposal by electroflotation. Chemical Engineering Journal, v. 34, n. 28. p, 503-509. 2007.

3. CERQUEIRA, A.A., MARQUES, M.R.C. Avaliação do processo eletrolítico em corrente alternada no tratamento de água de produção. Química nova, v. 34, n. 1, p. 59-63. 2011.

4. COMNINELLIS, C., PULGARIN, C. Anodic oxidation of phenol for wastewater treatment. Journal of Appl. Electrochemical, n. 21, p. 703-708, 1991.

5. FREIRE, D.D.C., SANT'ANNA JR., G.L. A proposed method modification for the determination of COD in saline waters. Environmental Technology, v. 19, p. 1243-1247, 1998

6. KIM, T.H., PARK, C., LEE, J., SHIN, E.B., KIM, S. Pilot scale treatment of textile wastewater by combined process (fluidized biofilm process-chemical coagulation-electrochemical oxidation). Water research, v. 36, n. 1, p. 3979-3988, 2002.

7. NAHUI, F. N. B., NASCIMENTO, M. R., CAVALCANTI, E. B., VILAR, E. O. Electroflotation of Emulsified Oil in Industrial Wastes Evaluated with a Full Factorial Design. Brazilian Journal of Chemical engineering, v. 3, n. 25, p. 435-442, 2008.

8. RAJKUMAR, D., PALANIVELU, K. Electrochemical treatment of industrial wastewater. Journal of hazardous materials, v. 13. n. 1. p.. 123-129. 2004.

9. QUINETE, N. S. Extração de poluentes organoclorados persistentes em fragmentos remanescentes da mata atlântica, rj: comparação de métodos. Dissertação de Mestrado. Universidade Federal Fluminense. 2005.

10. SANTOS, E.P., DUTRA, A.J.B. Concentração de partículas minerais finas e ultrafinas por eletroflotação, holos, v. 5, n. 1, p. 20-29. 2011. 